\title{
ОБ ОДНОМ МЕТОДЕ ПОСТРОЕНИЯ ЛОКАЛЬНЫХ ПАРАБОЛИЧЕСКИХ СПЛАЙНОВ С ДОПОЛНИТЕЛЬНЫМИ УЗЛАМИ
}

\author{
Ю. Н. Субботин, В. Т. Шевалдин
}

\begin{abstract}
В работе предложен общий метод построения локальных параболических сплайнов для функций, заданных сеточно на числовой оси или на отрезке оси, с произвольным расположением узлов. Частными случаями этой схемы являются сплайны Ю.Н. Субботина и Б. И. Квасова. Для сплайнов Квасова рассмотрены иные граничные условия, чем у Квасова, и исследованы их аппроксимативные и сглаживающие свойства в случае, когда узлы сплайна расположены равномерно. В частности, найдены двусторонние оценки погрешности аппроксимации классов функций $W_{\infty}^{2}$ и $W_{\infty}^{3}$ такими сплайнами в равномерной метрике и вычислены точно равномерные константы Лебега и нормы вторых производных на классе $W_{\infty}^{2}$. Произведено сравнение этих свойств со сплайнами Субботина.

Ключевые слова: локальные параболические сплайны, аппроксимация, интерполяция, равномерные узлы.

Yu. N. Subbotin, V.T. Shevaldin. A method of construction of local parabolic splines with additional knots.

We propose a general method for the construction of local parabolic splines with an arbitrary arrangement of knots for functions given on grid subsets of the numerical axis or its segment. Special cases of this scheme are Yu. N. Subbotin's and B. I. Kvasov's splines. For Kvasov's splines, we consider boundary conditions different from those suggested by Kvasov. We study the approximating and smoothing properties of these splines in the case of uniform knots. In particular, we find two-sided estimates of the error of approximation of the function classes $W_{\infty}^{2}$ and $W_{\infty}^{3}$ by these splines in the uniform metric and calculate the exact uniform Lebesgue constants and the norms of the second derivatives on the class $W_{\infty}^{2}$. These properties are compared with the corresponding properties of Subbotin's splines.
\end{abstract}

Keywords: local parabolic splines, approximation, interpolation, equally spaced knots.

MSC: 41A15

DOI: $10.21538 / 0134-4889-2019-25-2-205-219$

\section{Введение}

Авторами статьи (см. [1;2]) были построены локальные параболические сплайны (для функций, заданных на оси или на отрезке оси), сохраняющие линейные функции, с произвольным расположением узлов, обладающие хорошими аппроксимативными свойствами и свойствами локального сохранения знака, монотонности и выпуклости аппроксимируемых функций (см., например, [3]). Эти сплайны и их обобщения нашли широкое применение в задачах вычислительной математики (см., например, [4-6]). В настоящей работе приведена общая схема построения подобных конструкций, частным случаем которой являются сплайны из работ $[1 ; 2]$. В статье более подробно исследуется другая конструкция локальных параболических сплайнов, вытекающая из общей схемы, причем исследуемые сплайны построены как для функций, заданных на оси, так и для функций, определенных только на отрезке (с определенным выбором граничных условий в концах отрезка). Ю. С. Волков заметил, что описываемая далее конструкция этих сплайнов уже встретилась в работе Б. И. Квасова [7, формулы 1.1 и 3.1], но там исследуются сплайны с другими граничными условиями. В отличие от широко известных схем построения локальных сплайнов (см. [8-10]) параболические сплайны Квасова интерполируют значения аппроксимируемой функции в основных узлах сплайна (при этом дополнительные узлы выбираются в серединах отрезков между соседними основными). 
Кроме того, в настоящей работе на классах дифференцируемых функций исследуются аппроксимативные и сглаживающие свойства построенных сплайнов в случае, когда узлы сплайна расположены равномерно, а также произведено сравнение свойств отмеченных конструкций.

\section{1. Общий подход}

Рассмотрим на оси $\mathbb{R}$ бесконечную в обе стороны сетку узлов

$$
\cdots<x_{-1}<x_{0}<x_{1}<x_{2}<\cdots, \quad h_{j}=x_{j+1}-x_{j} \quad(j \in \mathbb{Z}) .
$$

Для функции $f: \mathbb{R} \rightarrow \mathbb{R}$ обозначим

$$
\Delta^{2} y_{j}=\left[y_{j}, y_{j+1}, y_{j+2}\right]=f\left[x_{j}, x_{j+1}, x_{j+2}\right]=\frac{y_{j+2}}{h_{j+1}\left(h_{j}+h_{j+1}\right)}-\frac{y_{j+1}}{h_{j} h_{j+1}}+\frac{y_{j}}{h_{j}\left(h_{j}+h_{j+1}\right)} \quad(j \in \mathbb{Z})
$$

- вторую разделенную разность функции $f$ по ее значениям $y_{j}=f\left(x_{j}\right)$ в точках $x_{j}, x_{j+1}, x_{j+2}$. Параболический сплайн $S$ на отрезке $\left[x_{j} ; x_{j+1}\right](j \in \mathbb{Z})$ будем строить в виде

$$
S(x)=S(f, x)=\alpha_{j}+\beta_{j}\left(x-x_{j}\right)+\gamma_{j}\left(x-x_{j}\right)^{2}+\delta_{j}\left(\left(x-x_{j+1 / 2}\right)_{+}\right)^{2},
$$

где $t_{+}=\max \{0 ; t\}, x_{j+1 / 2}=(1 / 2)\left(x_{j}+x_{j+1}\right)$, а коэффициенты $\alpha_{j}, \beta_{j}, \gamma_{j}, \delta_{j} \in \mathbb{R}$ будем находить из четырех условий $(j \in \mathbb{Z})$ :

1) $S\left(x_{j}\right)=y_{j}+k_{j} \Delta^{2} y_{j-1}$,

2) $S\left(x_{j+1}\right)=y_{j+1}+k_{j+1} \Delta^{2} y_{j}$,

3) $\left.S^{\prime \prime}\right|_{x \in\left(x_{j} ; x_{j+1 / 2}\right)}=A_{j} \Delta^{2} y_{j-1}+B_{j} \Delta^{2} y_{j}$,

4) $\left.S^{\prime \prime}\right|_{x \in\left(x_{j+1 / 2} ; x_{j+1}\right)}=C_{j} \Delta^{2} y_{j-1}+D_{j} \Delta^{2} y_{j}$,

где числа $k_{j}, k_{j+1}, A_{j}, B_{j}, C_{j}, D_{j} \in \mathbb{R}$ (об их выборе см. дальнейшие выкладки). Решая систему (1.2), выводим следующие равенства:

$$
\begin{aligned}
& \alpha_{j}=y_{j}+k_{j} \Delta^{2} y_{j-1}, \quad 2 \gamma_{j}=A_{j} \Delta^{2} y_{j-1}+B_{j} \Delta^{2} y_{j}, \\
& 2 \delta_{j}=\left(C_{j}-A_{j}\right) \Delta^{2} y_{j-1}+\left(D_{j}-B_{j}\right) \Delta^{2} y_{j}, \\
& \beta_{j}=\frac{y_{j+1}-y_{j}}{h_{j}}+\frac{k_{j+1} \Delta^{2} y_{j}-k_{j} \Delta^{2} y_{j-1}}{h_{j}}-\frac{h_{j}}{8}\left[\left(3 A_{j}+C_{j}\right) \Delta^{2} y_{j-1}+\left(3 B_{j}+D_{j}\right) \Delta^{2} y_{j}\right] .
\end{aligned}
$$

Потребуем, чтобы функция $S$, определенная формулой (1.1), удовлетворяла условию

$$
S^{\prime}\left(x_{j+1}-0\right)=S^{\prime}\left(x_{j+1}+0\right) \quad(j \in \mathbb{Z}) .
$$

Равенство (1.4) с учетом (1.3) равносильно уравнению

$$
\begin{gathered}
\left(h_{j}+h_{j+1}\right) \Delta^{2} y_{j}+\left[\frac{k_{j+2}}{h_{j+1}}-\frac{h_{j+1}}{8}\left(3 B_{j+1}+D_{j+1}\right)\right] \Delta^{2} y_{j+1} \\
+\left[\frac{k_{j}}{h_{j}}+\frac{h_{j}}{8}\left(3 A_{j}+C_{j}\right)\right] \Delta^{2} y_{j-1}+\left[-\frac{k_{j+1}}{h_{j+1}}-\frac{h_{j+1}}{8}\left(3 A_{j+1}+C_{j+1}\right)-\frac{k_{j+1}}{h_{j}}+\frac{h_{j}}{8}\left(3 B_{j}+D_{j}\right)\right] \Delta^{2} y_{j} \\
=h_{j}\left(\frac{A_{j}+C_{j}}{2} \Delta^{2} y_{j-1}+\frac{D_{j}+B_{j}}{2} \Delta^{2} y_{j}\right)
\end{gathered}
$$


и означает, что $S \in C^{1}(\mathbb{R})$. В последнем равенстве приравняем коэффициенты при разделенных разностях $\Delta^{2} y_{j-1}, \Delta^{2} y_{j}$ и $\Delta^{2} y_{j+1}$ в левой и правой части. Получим систему из трех разностных уравнений

$$
\left\{\begin{array}{l}
\frac{k_{j+2}}{h_{j+1}}=\frac{h_{j+1}}{8}\left(3 B_{j+1}+D_{j+1}\right), \\
\frac{k_{j}}{h_{j}}=\frac{h_{j}}{8}\left(A_{j}+3 C_{j}\right) \\
h_{j+1}+h_{j}-\frac{k_{j+1}}{h_{j+1}}-\frac{k_{j+1}}{h_{j}}=\frac{h_{j+1}}{8}\left(3 A_{j+1}+C_{j+1}\right)+\frac{h_{j}}{8}\left(B_{j}+3 D_{j}\right) .
\end{array}\right.
$$

В двух последующих разделах выделены два частных случая решения системы (1.5).

\section{2. Случай Ю. Н. Субботина формосохраняющей аппроксимации}

Положим $B_{j}=C_{j}=0(j \in \mathbb{Z})$. Тогда из (1.5) выводим следующие равенства:

$$
k_{j}=\frac{h_{j-1} h_{j}}{4}, \quad A_{j}=\frac{2 h_{j-1}}{h_{j}}, \quad D_{j}=\frac{2 h_{j+1}}{h_{j}} \quad(j \in \mathbb{Z}) .
$$

Сплайн $S \in C^{1}(\mathbb{R})$ с такими параметрами может быть записан в виде

$$
\begin{gathered}
S(x)=S(f, x)=y_{j}+\frac{h_{j-1} h_{j}}{4} \Delta^{2} y_{j-1}+\frac{y_{j+1}-y_{j-1}}{h_{j}+h_{j-1}}\left(x-x_{j}\right) \\
+\frac{h_{j-1}}{h_{j}}\left(x-x_{j}\right)^{2} \Delta^{2} y_{j-1}+\left(\frac{h_{j+1}}{h_{j}} \Delta^{2} y_{j}-\frac{h_{j-1}}{h_{j}} \Delta^{2} y_{j-1}\right)\left(\left(x-x_{j+1 / 2}\right)_{+}^{0}\right)^{2}, \\
x \in\left[x_{j} ; x_{j+1}\right] \quad(j \in \mathbb{Z}) .
\end{gathered}
$$

В силу построения (см. переход к равенствам (1.5)) сплайн вида (2.1) сохраняет линейные функции (но не квадратичные) в том смысле, что

$$
S(1, \cdot)=1, \quad S(x, \cdot)=x
$$

но не является интерполяционным, т. е. не удовлетворяет условию

$$
S\left(x_{j}\right)=y_{j} \quad(j \in \mathbb{Z}),
$$

поскольку $k_{j}>0(j \in \mathbb{Z})$. При $h_{j}=h$ (т. е. в случае равномерной сетки узлов) сплайны $S$ вида (2.1) появились и изучались в работе [1] первого автора, а в случае произвольного расположения узлов - позже в работе [2] второго автора. Данный раздел в основном посвящен изложению формосохраняющих и аппроксимативных свойств этих сплайнов.

Прежде всего отметим, что в общем случае (т. е. для произвольной сетки узлов) построенный на всей числовой оси сплайн $S$ вида (2.1) реализует трехточечную схему локальной аппроксимации, поскольку на любом отрезке вида $\left[x_{j-1 / 2} ; x_{j+1 / 2}\right](j \in \mathbb{Z})$ он зависит только от трех значений $y_{j-1}, y_{j}$ и $y_{j+1}$ аппроксимируемой функции $f$. Формула $(2.1)$ задает линейный положительный оператор, действующий из пространства $C$ непрерывных на всей числовой оси функций $f$ в пространство непрерывно дифференцируемых функций $C^{1}$. Положительность оператора $S$ понимается в следующем смысле: если $f(x) \geq 0(x \in \mathbb{R})$, то $S(f, x) \geq 0(x \in \mathbb{R})$. Иными словами, имеет место сохранение сплайном $S$ знака аппроксимируемой функции. Напомним, что величина

$$
L=\|S\|_{C}^{C}=\sup _{\|f\|_{C} \leq 1}\|S(f, \cdot)\|_{C}
$$


является нормой (равномерной) оператора $S$ и называется константой Лебега метода $S$. Для сплайнов $S$ вида (2.1) в [3, теорема 5.7] доказано, что $L=1$. Следующая теорема устанавливает другие формосохраняющие свойства сплайнов $S$ вида (2.1).

Теорема $A\left[1\right.$, случай $h_{j}=h$, предложение; 2 , теорема 1]. Сплайн $S$ наследует локально свойство монотонности исходных данных $\left\{y_{j}\right\}_{j \in \mathbb{Z}}$ в том смысле, ито

а) если $y_{j+1} \geq y_{j} \geq y_{j-1}\left(\right.$ или $\left.y_{j+1} \leq y_{j} \leq y_{j-1}\right)$, то функиия $S$ не убъвает (не возрастает) на промежутке $\left(x_{j} ; x_{j+1 / 2}\right)$;

б ) если $y_{j+2} \geq y_{j+1} \geq y_{j}\left(\right.$ или $\left.y_{j+2} \leq y_{j+1} \leq y_{j}\right)$, то функиия $S$ не убывает (не возрастает) на промежутке $\left(x_{j+1 / 2} ; x_{j+1}\right)$.

Сплайн $S$ наследует локально свойство выпуклости исходных данных $\left\{y_{j}\right\}_{j \in \mathbb{Z}}$, а именно:

а) если $\Delta^{2} y_{j-1} \geq 0\left(\Delta^{2} y_{j-1} \leq 0\right)$, то функиия $S$ выпукла вниз (вверх) на промежутке $\left(x_{j} ; x_{j+1 / 2}\right)$,

б) если $\Delta^{2} y_{j} \geq 0\left(\Delta^{2} y_{j} \leq 0\right)$, то функиия $S$ выпукла вниз (вверх) на промежутке $\left(x_{j+1 / 2}\right.$; $\left.x_{j+1}\right)$.

Описанная конструкция сплайнов была развита авторами $[1 ; 2]$ и для функций $f$, заданных своими значениями только на отрезке числовой оси. Кроме того, эта схема была обобщена и на другие виды сплайнов: экспоненциальные, тригонометрические, интерполяционные в среднем и т. д. (см., например, [3, гл. 2-5]).

Для того, чтобы сформулировать аппроксимативные свойства сплайна $S$, дадим несколько определений. Пусть, как обычно, $A C$ - класс локально абсолютно непрерывных функций, $L_{\infty}$ - класс функций $f$, существенно ограниченных на $\mathbb{R}$, с обычным определением нормы

$$
\|f\|_{\infty}=\|f\|_{L_{\infty}}=\operatorname{ess}_{x \in \mathbb{R}}|f(x)|
$$

и

$$
W_{\infty}^{2}=W_{\infty}^{2}(\mathbb{R})=\left\{f: f^{\prime} \in A C,\left\|f^{\prime \prime}\right\|_{\infty} \leq 1\right\}
$$

- соболевский класс дважды дифференцируемых почти всюду функций. В следующей теореме мы ограничимся только случаем равномерной сетки узлов сплайна $S$ (т. е. положим $h_{j}=h$ ).

Теорема $B$ [1, теоремы 1, 3, формула (1.14)] Имеют место следующие равенства:

$$
\sup _{f \in W_{\infty}^{2}}\|f-S\|_{C}=\frac{h^{2}}{8}, \quad \sup _{f \in W_{\infty}^{2}}\left\|f^{\prime}-S^{\prime}\right\|=\frac{h}{2}, \quad \sup _{f \in W_{\infty}^{2}}\left\|S^{\prime \prime}\right\|_{\infty}=1 .
$$

Для произвольной сетки узлов более общее утверждение доказано вторым автором в [2]. Поясним равенства из теоремы $B$. Они означают, что для периодических функций $f$ метод $(2.1)$ локальной аппроксимации сплайнами (и их производными) оказался оптимальным в смысле поперечников по Колмогорову и по Коновалову, а именно, для класса функций $W_{\infty}^{2}$ было найдено еще одно (третье по счету) наилучшее экстремальное подпространство (помимо тригонометрических полиномов и интерполяционных параболических сплайнов с равномерными узлами). Соответствующие определения и историю вопроса см., например, в [1]. Отметим, что статья [1] породила целый цикл последующих работ Ю. Н. Субботина и С. А. Теляковского по поперечникам по Коновалову (их еще называют относительными) классов дифференцируемых функций и оценкам констант Лебега интерполяционных сплайнов и тригонометрическмх полиномов.

\section{3. Случай интерполяционных сплайнов Б. И. Квасова}

Положим $k_{j}=0$ для любого $j \in \mathbb{Z}$. В этом случае сплайн $S$ вида (1.1), параметры которого удовлетворяют системе уравнений (1.5), в силу (1.2) является интерполяционным в том смысле, что

$$
S\left(x_{j}\right)=y_{j} \quad(j \in \mathbb{Z}) .
$$


Его узлы в дальнейшем будем называть основными, а узлы $\left\{x_{j+1 / 2}\right\}-$ дополнителъными. В этом случае система уравнений (1.5) равносильна следующей:

$$
\left\{\begin{array}{l}
3 B_{j}+D_{j}=0 \\
A_{j}+3 C_{j}=0 \\
h_{j}\left(B_{j}+1\right)+h_{j+1}\left(C_{j+1}+1\right)=0
\end{array} \quad(j \in \mathbb{Z}) .\right.
$$

Таким образом, для определения параметров $A_{j}, B_{j}, C_{j}$ и $D_{j}$ сплайна $S$ имеется система из трех линейных уравнений. Мы положим $B_{j}=-1$ (данный выбор обоснуем чуть позже). Возможно, существуют и другие интересные случаи выбора параметров в системе (3.1). При $B_{j}=-1(j \in \mathbb{Z})$ однозначно выводим равенства: $C_{j}=-1, A_{j}=D_{j}=3(j \in \mathbb{Z})$. При этом сплайн $S$ на всей оси $\mathbb{R}$ можно записать в виде

$$
\begin{aligned}
S(x) & =y_{j}+\left(x-x_{j}\right)\left[\frac{y_{j+1}-y_{j}}{h_{j}}-h_{j} \Delta^{2} y_{j-1}\right]+\frac{\left(x-x_{j}\right)^{2}}{2}\left(3 \Delta^{2} y_{j-1}-\Delta^{2} y_{j}\right) \\
& +\left(\left(x-x_{j+1 / 2}\right)_{+}\right)^{2}\left(2 \Delta^{2} y_{j}-2 \Delta^{2} y_{j-1}\right), \quad x \in\left[x_{j}, x_{j+1}\right] \quad(j \in \mathbb{Z}) .
\end{aligned}
$$

В силу построения (см. переход к равенствам (1.5)) сплайн $S \in C^{1}(\mathbb{R})$ и сохраняет линейные функции, т. е.

$$
S(1, \cdot)=1, \quad S(x, \cdot)=x .
$$

Но оказывается, что выбор $B_{j}=-1$ приводит к равенству

$$
S\left(x^{2}, \cdot\right)=x^{2} .
$$

Это равенство следует непосредственно из формулы (3.2). Значит, интерполяционный сплайн вида (3.2) сохраняет пространство алгебраических многочленов второй степени.

Ясно также, что $S$ реализует четырехточечную схему локальной аппроксимации, поскольку на любом отрезке $\left[x_{j} ; x_{j+1}\right](j \in \mathbb{Z})$ он зависит только от четырех значений $y_{j-1}, y_{j}, y_{j+1}$ и $y_{j+2}$ аппроксимируемой функции $f$. Покажем, как можно уточнить предложенную схему построения аппроксимирующего сплайна $S$, если исходная функция $f$ задана только на отрезке числовой оси.

Не ограничивая общности, рассмотрим отрезок $[0 ; a]$ и сетку узлов $0=x_{0}<x_{1}<x_{2}<$ $\cdots<x_{n-1}<x_{n}=a$ на нем, и пусть $y_{j}=f\left(x_{j}\right)(j=\overline{0, n})$. На отрезках $\left[x_{j} ; x_{j+1}\right](j=\overline{1, n-2})$ сплайн $S$ строим по формуле (3.2). На первом отрезке $\left[0 ; x_{1}\right]$ разбиения функцию $S$ будем искать в виде

$$
S(x)=a_{1}+a_{2} x+a_{3}\left(\left(x-\frac{h_{0}}{2}\right)_{+}\right)^{2},
$$

а числа $a_{1}, a_{2}$ и $a_{3}$ найдем из условий

$$
S(0)=y_{0}, \quad S\left(x_{1}\right)=y_{1}, \quad S^{\prime}\left(x_{1}-0\right)=S^{\prime}\left(x_{1}+0\right) .
$$

Заметим, что из (3.2) следует равенство $S^{\prime}\left(x_{1}+0\right)=\frac{y_{1}-y_{0}}{h_{0}}+h_{0} \Delta^{2} y_{0}$. Поэтому для чисел $a_{1}, a_{2}$ и $a_{3}$ выводим формулы

$$
a_{1}=y_{0}, \quad a_{2}=\frac{y_{1}-y_{0}}{h_{0}}-\frac{h_{0}}{3} \Delta^{2} y_{0}, \quad a_{3}=\frac{4}{3} \Delta^{2} y_{0} .
$$

Аналогично на последнем отрезке $\left[a-h_{n-1} ; a\right]=\left[x_{n-1} ; x_{n}\right]$ разбиения функцию $S$ строим в виде

$$
S(x)=b_{1}+b_{2}(a-x)+b_{3}\left(\left(x-a+\frac{h_{n-1}}{2}\right)_{-}\right)^{2},
$$

где функция-"срезка" $t_{-}=\min \{t, 0\}$, а числа $b_{1}, b_{2}$ и $b_{3}$ находятся из равенств

$$
S(a)=y_{n}, \quad S\left(a-h_{n-1}\right)=y_{n-1}, \quad S^{\prime}\left(a-h_{n-1}-0\right)=S^{\prime}\left(a-h_{n-1}+0\right) .
$$


Из (3.2) следует, что

$$
S^{\prime}\left(a-h_{n-1}-0\right)=\frac{y_{n}-y_{n-1}}{h_{n-1}}-h_{n-1} \Delta^{2} y_{n-2} .
$$

Поэтому

$$
b_{1}=y_{n}, \quad b_{2}=\frac{y_{n-1}-y_{n}}{h_{n-1}}-\frac{h_{n-1}}{3} \Delta^{2} y_{n-2}, \quad b_{3}=\frac{4}{3} \Delta^{2} y_{n-2} .
$$

Таким образом,

$$
\begin{gathered}
S(x)=y_{0}+\left[\frac{y_{1}-y_{0}}{h_{0}}-\frac{h_{0}}{3} \Delta^{2} y_{0}\right] x+\frac{4}{3} \Delta^{2} y_{0}\left(\left(x-\frac{h_{0}}{2}\right)_{+}\right)^{2}, \quad x \in\left[0 ; x_{1}\right], \\
S(x)=y_{n}+\left[\frac{y_{n-1}-y_{n}}{h_{n-1}}-\frac{h_{n-1}}{3} \Delta^{2} y_{n-2}\right](a-x)+\frac{4}{3} \Delta^{2} y_{n-2}\left(\left(x-a+\frac{h_{n-1}}{2}\right)_{-}\right)^{2}, \quad x \in\left[x_{n-1} ; a\right] .
\end{gathered}
$$

Равенства (3.2) при $j=\overline{1, n-2}$ и (3.3) позволяют утверждать, что построенная функция $S(x)=S(f, x) \in C^{1}[0 ; a]$ является линейной на отрезках $\left[0 ; x_{1} / 2\right]$ и $\left[x_{n-1 / 2} ; a\right]$ и интерполирует функцию $f:[0 ; a] \rightarrow \mathbb{R}$ во всех основных узлах $\left\{x_{j}\right\}_{j=0}^{n}$ сетки на отрезке $[0 ; a]$.

3 а м е ч а н и е 1. Поясним, почему только что описанный второй случай решения системы (1.5) естественно называть случаем сплайнов Б. И. Квасова. Дело в том, что формула (3.2) локальных сплайнов на оси $\mathbb{R}$ фактически следует из результатов его работы [7, формулы (1.1) и (3.1)], посвященной интерполяции функций эрмитовыми параболическими сплайнами, в которой локальный параболический сплайн с основными узлами в точках $\left\{x_{j}\right\}$ и дополнительными - в точках $\left\{x_{j+1 / 2}\right\}$ на отрезке [0; $\left.a\right]$ строится по значениям функции $f\left(x_{j}\right)(j=\overline{0, n})$ и ее производных $f^{\prime}\left(x_{j}\right)(j=\overline{0, n})$, а затем значения производных $f^{\prime}\left(x_{j}\right)(j=\overline{0, n})$ заменяются некоторыми комбинациями разделенных разностей первого порядка.

\section{4. Интерполяционные локальные сплайны с равноотстоящими узлами}

В данном разделе исследуются свойства (константы Лебега, нормы вторых производных, величины аппроксимации) сплайнов, заданных формулами (3.2) и (3.3) в случае, когда узлы сплайна $\left\{x_{j}\right\}$ расположены равномерно (т. е. в случае $h_{j}=h(j \in \mathbb{Z})$ ). В случае произвольных узлов подобные исследования приводят к громоздким выкладкам.

Из (3.2) при $h_{j}=h$ выводим равенство

$$
\begin{gathered}
S(x)=y_{j}+\frac{x-j h}{2 h}\left(y_{j+1}-y_{j-1}\right)+\frac{(x-j h)^{2}}{4 h^{2}}\left(-y_{j+2}+5 y_{j+1}-7 y_{j}+3 y_{j-1}\right) \\
+\frac{\left((x-(j+1 / 2) h)_{+}\right)^{2}}{h^{2}}\left(y_{j+2}-3 y_{j+1}+3 y_{j}-y_{j-1}\right), \quad x \in[j h ;(j+1) h] \quad(j \in \mathbb{Z}),
\end{gathered}
$$

где $y_{j}=f(j h)$.

При дальнейшем исследовании свойств сплайна $S$ в формуле $(4.1)$ на отрезке $[j h ;(j+1) h]$ можно, не ограничивая общности, считать $j=0$ и рассматривать только отрезок $[0 ; h]$. Более того, покажем, что на самом деле сплайн $S$ обладает определенной “симметрией” относительно середины любого отрезка $[j h ;(j+1) h]$ и поэтому при доказательствах равномерных оценок погрешности аппроксимации достаточно ограничиваться только случаем $x \in[0 ; h / 2]$.

Из (4.1) при $j=0$ имеем

$$
\begin{array}{ll}
S(x)=C_{2}(x) y_{2}+C_{1}(x) y_{1}+C_{0}(x) y_{0}+C_{-1}(x) y_{-1}, & x \in\left[0 ; \frac{h}{2}\right], \\
S(x) & =\bar{C}_{2}(x) y_{2}+\bar{C}_{1}(x) y_{1}+\bar{C}_{0}(x) y_{0}+\bar{C}_{-1}(x) y_{-1}, \quad x \in\left[\frac{h}{2} ; h\right],
\end{array}
$$


причем

$$
\begin{aligned}
& \bar{C}_{2}(x)=\frac{3 x^{2}}{4 h^{2}}-\frac{x}{h}+\frac{1}{4}=C_{-1}(h-x), \\
& \bar{C}_{1}(x)=-\frac{7 x^{2}}{4 h^{2}}+\frac{7 x}{2 h}-\frac{3}{4}=C_{0}(h-x), \\
& \bar{C}_{0}(x)=\frac{5 x^{2}}{4 h^{2}}-\frac{3 x}{h}+\frac{7}{4}=C_{1}(h-x), \\
& \bar{C}_{-1}(x)=-\frac{x^{2}}{4 h^{2}}+\frac{x}{2 h}-\frac{1}{4}=C_{2}(h-x) .
\end{aligned}
$$

Из выписанных формул следует "симметричность" сплайна $S$ на отрезке $[0 ; h]$ относительно его середины в том смысле, что если заменить интерполируемые значения функции $y_{2}, y_{1}, y_{0}$ и $y_{-1}$ соответственно на $y_{-1}, y_{0}, y_{1}$ и $y_{2}$ и коэффициенты при них по формулам (4.3), то первое равенство в (4.2) перейдет во второе и наоборот. Поэтому при доказательстве следующих утверждений (см. дальше теоремы $1-3)$ будем ограничиваться только случаем $x \in[0 ; h / 2]$.

Сплайн $S$ вида (4.1) задает линейный оператор, ставящий в соответствие каждой непрерывной функции $f: \mathbb{R} \rightarrow \mathbb{R}$ непрерывно дифференцируемый сплайн $S(f, x)$. Нас интересует константа Лебега

$$
L=\sup _{\|f\|_{C} \leq 1}\|S(f, \cdot)\|_{C}
$$

этого оператора.

Теорема 1. Имеет место следующее равенство: $L=\frac{5}{4}$.

Д о к а з а т е л ь с т в о. Не ограничивая общности, положим $j=0$ и пусть $x \in[0 ; h / 2]$. Из (4.2), (4.3) имеем

$$
S(x)=\sum_{k=-1}^{2} C_{k}(x) y_{k},
$$

где

$$
\begin{gathered}
C_{-1}(x)=-\frac{x}{2 h}+\frac{3 x^{2}}{4 h^{2}} \leq 0, \quad C_{0}(x)=1-\frac{7 x^{2}}{4 h^{2}} \geq 0 \\
C_{1}(x)=\frac{x}{2 h}+\frac{5 x^{2}}{4 h^{2}} \geq 0, \quad C_{2}(x)=-\frac{x^{2}}{4 h^{2}} \leq 0 \quad\left(x \in\left[0 ; \frac{h}{2}\right]\right) .
\end{gathered}
$$

Значит, при $\left|y_{k}\right| \leq 1(k=\overline{-1,2})$ имеет место оценка

$|S(x)| \leq \max _{k=-1,2}\left|y_{k}\right|\left(\frac{x}{2 h}-\frac{3 x^{2}}{4 h^{2}}+1-\frac{7 x^{2}}{4 h^{2}}+\frac{x}{2 h}+\frac{5 x^{2}}{4 h^{2}}+\frac{x^{2}}{4 h^{2}}\right)=\max _{k=-1,2}\left|y_{k}\right| \max _{x \in[0 ; h / 2]}\left(1+\frac{x}{h}-\frac{x^{2}}{h^{2}}\right)=\frac{5}{4}$.

Выписанная поточечная оценка сверху для $|S(x)|$ является точной, поскольку она достигается при $x=h / 2$ для последовательности значений $y_{-1}=y_{2}=-1, y_{0}=y_{1}=1$.

Теорема 1 доказана.

Теорема 2. Для сплайнов $S(x)=S(f, x)$, заданных формулой (4.1), имеет место следующее равенство:

$$
\sup _{f \in W_{\infty}^{2}}\left\|S^{\prime \prime}\right\|_{\infty}=\frac{13}{8} .
$$

Д о к а з а т е л в с т в о. Аппроксимируемую функцию $f$ запишем в виде

$$
f(x)=f(j h)+f^{\prime}(j h)(x-j h)+\int_{j h}^{x}(x-t) f^{\prime \prime}(t) d t .
$$


Тогда из (4.1) при $x \in[j h ;(j+1 / 2) h]$ имеем

$$
\begin{gathered}
S(x)=f(j h)+\frac{x-j h}{2 h}\left[f(j h)+f^{\prime}(j h) h+\int_{j h}^{(j+1) h}((j+1) h-t) f^{\prime \prime}(t) d t\right. \\
\left.-f(j h)+f^{\prime}(j h) h-\int_{j h}^{(j-1) h}((j-1) h-t) f^{\prime \prime}(t) d t\right] \\
+\frac{(x-j h)^{2}}{4 h^{2}}\left[-f(j h)-2 f^{\prime}(j h) h-\int_{j h}^{(j+2) h}((j+2) h-t) f^{\prime \prime}(t) d t+5 f(j h)+5 f^{\prime}(j h) h\right. \\
\left.+5 \int_{j h}^{(j+1) h}((j+1) h-t) f^{\prime \prime}(t) d t-7 f(j h)+3 f(j h)-3 f^{\prime}(j h) h+3 \int_{j h}^{(j-1) h}((j-1) h-t) f^{\prime \prime}(t) d t\right] .
\end{gathered}
$$

Не ограничивая общности, положим, что $j=0$. Тогда

$$
S^{\prime \prime}(x)=\int_{-h}^{0} L_{1}(t) f^{\prime \prime}(t) d t+\int_{h}^{0} L_{2}(t) f^{\prime \prime}(t) d t+\int_{h}^{2 h} L_{3}(t) f^{\prime \prime}(t) d t
$$

где

$$
\begin{gathered}
L_{1}(t)=\frac{3(h+t)}{2 h^{2}} \geq 0 \quad(-h \leq t \leq 0), \quad L_{2}(t)=\frac{3 h-4 t}{2 h^{2}} \quad(0 \leq t \leq h), \\
L_{3}(t)=-\frac{2 h-t}{2 h^{2}} \leq 0 \quad(h \leq t \leq 2 h) .
\end{gathered}
$$

Ядро $L_{2}(t)$ меняет знак один раз в точке $t=3 h / 4$ с плюса на минус. Значит, на классе функций $W_{\infty}^{2}$ справедлива точная оценка сверху

$$
\left\|S^{\prime \prime}\right\|_{\infty} \leq \int_{-h}^{0} L_{1}(t) d t+\int_{0}^{3 h / 4} L_{2}(t) d t-\int_{3 h / 4}^{h} L_{2}(t) d t-\int_{h}^{2 h} L_{3}(t) d t=\frac{13}{8},
$$

которая достигается, если $f^{\prime \prime}(t)=1(t \in[-h ; 3 h / 4])$ и $f^{\prime \prime}(t)=-1(t \in(3 h / 4 ; 2 h])$.

Теорема 2 доказана.

Теорема 3. Для сплайнов $S(x)=S(f, x)$, определенных формулой (4.1), имеет место следующее равенство:

$$
\sup _{f \in W_{\infty}^{2}}\|f-S\|_{C}=K h^{2},
$$

где $K$ - некоторая константа, причем $9 / 64 \leq K \leq 5 / 32$.

Д о к а з а т е л ь с т в о. При доказательстве теоремы 3 будем использовать представление сплайна $S$ в форме (4.5) (при этом функцию $f$ представим в виде (4.4)). Заметим, что в разности $S(x)-f(x)$ при использовании этих формул будут отсутствовать неинтегральные слагаемые (они взаимно уничтожаются), так как $S(f, x)$ сохраняет линейные функции. Значит, при $j=0$ и $0 \leq x \leq h / 2$ имеет место следующая формула:

$$
S(x)-f(x)=\int_{-h}^{0} K_{1}(x, t) f^{\prime \prime}(t) d t+\int_{0}^{x} K_{2}(x, t) f^{\prime \prime}(t) d t+\int_{x}^{h} K_{3}(x, t) f^{\prime \prime}(t) d t+\int_{h}^{2 h} K_{4}(x, t) f^{\prime \prime}(t) d t,
$$


где

$$
\begin{aligned}
& K_{1}(x, t)=\frac{x}{4 h^{2}}(h+t)(3 x-2 h), \\
& K_{2}(x, t)=K_{3}(x, t)-(x-t)=\frac{x}{2 h}\left(h-t+\frac{x}{2 h}(3 h-4 t)\right)-x+t, \\
& K_{3}(x, t)=\frac{x}{2 h}\left(h-t+\frac{x}{2 h}(3 h-4 t)\right), \\
& K_{4}(x, t)=\frac{x^{2}}{4 h^{2}}(t-2 h) .
\end{aligned}
$$

Легко проверяется, что $K_{1}(x, t) \leq 0$ при $-h \leq t \leq 0,0 \leq x \leq h / 2$ и $K_{4}(x, t) \leq 0$ при $h \leq t \leq 2 h$, $0 \leq x \leq h / 2$. Поскольку

$$
K_{2}(x, 0)=\frac{x}{4 h}(3 x-2 h) \leq 0, \quad K_{2}(x, x)=\frac{x}{4 h^{2}}\left(2 h^{2}+h x-4 x^{2}\right) \geq 0
$$

при $0 \leq x \leq h / 2$, то функция $K_{2}(\cdot, t)$ как линейная функция от аргумента $t$ меняет знак ровно один раз (с минуса на плюс) на отрезке $[0 ; x]$. С другой стороны,

$$
K_{3}(x, h)=-\frac{x^{2}}{4 h} \leq 0, \quad K_{3}(x, x)=K_{2}(x, x) \geq 0
$$

при $0 \leq x \leq h / 2$. Поэтому линейная функция $K_{3}(\cdot, t)$ тоже ровно один раз меняет знак на отрезке $[x ; h]$ (с плюса на минус). Элементарные вычисления показывают, что нулем функции $K_{2}(\cdot, t)$ является число

$$
t_{1}=t_{1}(x)=\frac{x h(3 x-2 h)}{4 x^{2}+2 h x-4 h^{2}}
$$

а нулем функции $K_{3}(\cdot, t)$ - число

$$
t_{2}=t_{2}(x)=\frac{h(2 h+3 x)}{2(h+2 x)}
$$

Пусть

$$
\begin{gathered}
\varphi(x)=-\int_{-h}^{0} K_{1}(x, t) d t-\int_{0}^{t_{1}(x)} K_{2}(x, t) d t+\int_{t_{1}(x)}^{x} K_{2}(x, t) d t \\
+\int_{x}^{t_{2}(x)} K_{3}(x, t) d t-\int_{t_{2}(x)}^{h} K_{3}(x, t) d t-\int_{h}^{2 h} K_{4}(x, t) d t .
\end{gathered}
$$

Из (4.6) и проведенного исследования ядер $K_{s}(x, t)(s=\overline{1,4})$ для любой функции $f \in W_{\infty}^{2}$ получаем поточечную оценку сверху погрешности аппроксимации, а именно,

$$
|S(x)-f(x)| \leq \varphi(x) \quad\left(0 \leq x \leq \frac{h}{2}\right)
$$

которая является точной в том смысле, что последнее неравенство обращается в равенство, если положить $f^{\prime \prime}(t)=-1\left(-h \leq t \leq t_{1}(x)\right), f^{\prime \prime}(t)=1\left(t_{1}(x)<t<t_{2}(x)\right), f^{\prime \prime}(t)=-1\left(t_{2}(x) \leq\right.$ $t \leq 2 h)$. Поскольку вычислить точно величину

$$
\|\varphi\|_{C[0 ; h / 2]}=\max _{x \in[0 ; h / 2]}|\varphi(x)|
$$

весьма сложно, то для доказательства теоремы 3 оценим последнюю величину сверху и снизу. Для оценки снизу положим $x=h / 2$, при этом $t_{1}=h / 8, t_{2}=7 h / 8$, и для функции $f$ такой, 
что

имеет место равенство

$$
f^{\prime \prime}(t)=\left\{\begin{array}{cl}
-1, & -h \leq t \leq \frac{h}{8} \\
1, & \frac{h}{8}<t<\frac{7 h}{8} \\
-1, & \frac{7 h}{8} \leq t \leq 2 h
\end{array}\right.
$$

$$
\left|S\left(\frac{h}{2}\right)-f\left(\frac{h}{2}\right)\right|=\frac{9 h^{2}}{64} .
$$

Для получения оценки сверху для $\|\varphi\|_{C[0 ; h / 2]}$ отметим, что из $(4.7),(4.8)$ следуют неравенства

$$
K_{2}(x, x) \geq\left|K_{2}(x, 0)\right|, \quad K_{3}(x, x)=K_{2}(x, x) \geq\left|K_{3}(x, h)\right| \quad\left(0 \leq x \leq \frac{h}{2}\right) .
$$

Значит,

$$
-\int_{0}^{t_{1}(x)} K_{2}(x, t) d t+\int_{t_{1}(x)}^{x} K_{2}(x, t) d t+\int_{x}^{t_{2}(x)} K_{3}(x, t) d t-\int_{t_{2}(x)}^{h} K_{3}(x, t) d t \leq \frac{h K_{2}(x, x)}{2}
$$

поскольку функции $K_{2}(\cdot, t)$ и $K_{3}(\cdot, t)$ линейны по $t$. Отсюда при любом $x \in[0 ; h / 2]$ получаем для функции $\varphi(x)$ оценку сверху

$$
\varphi(x) \leq-\int_{-h}^{0} K_{1}(x, t) d t+\frac{h K_{2}(x, x)}{2}-\int_{h}^{2 h} K_{4}(x, t) d t=\frac{h x-x^{2}}{4}+\frac{x}{8 h}\left(2 h^{2}+h x-4 x^{2}\right),
$$

в которой не участвуют числа $t_{1}(x)$ и $t_{2}(x)$. Наибольшее значение правой части $(4.9)$ равно $5 h^{2} / 32$ и достигается при $x=h / 2$. Таким образом, получено неравенство

$$
\frac{9}{64} h^{2} \leq \sup _{f \in W_{\infty}^{2}}\|f-S\|_{C} \leq \frac{5}{32} h^{2}
$$

Теорема 3 доказана.

Уточним эту теорему для локальных сплайнов, заданных формулами (3.2) и (3.3) на отрезке $[0 ; a]$. Для равномерной сетки узлов формулы (3.3) переписываются в следующем виде:

$$
\begin{gathered}
S(x)=y_{0}+\frac{1}{6 h}\left(-y_{2}+8 y_{1}-7 y_{0}\right) x+\frac{2}{3 h^{2}}\left(y_{2}-2 y_{1}+y_{0}\right)\left(\left(x-\frac{h}{2}\right)_{+}\right)^{2}, \quad(0 \leq x \leq h) \\
S(x)=y_{n}+\frac{1}{6 h}\left(-7 y_{n}+8 y_{n-1}-y_{n-2}\right)(a-x) \\
+\frac{2}{3 h^{2}}\left(y_{n}-2 y_{n-1}+y_{n-2}\right)\left(\left(x-a+\frac{h}{2}\right)_{-}\right)^{2}, \quad x \in[a-h ; a]
\end{gathered}
$$

Пусть $W_{\infty}^{2}[c ; d]=\left\{f: f^{\prime} \in A C,\left\|f^{\prime \prime}\right\|_{L_{\infty}[c ; d]} \leq 1\right\}$.

Теорема 4. Для сплайнов $S(x)=S(f, x)$, определенных формулой (4.1) nри $h \leq x \leq a-h$ и формулами (4.10) при $0 \leq x \leq h$ и $a-h \leq x \leq a$, имеют место следуюшие соотношения:

$$
\begin{gathered}
\sup _{f \in W_{\infty}^{2}[0 ; a]}\|f-S\|_{C[0 ; h / 2]}=\sup _{f \in W_{\infty}^{2}[0 ; a]}\|f-S\|_{C[a-h / 2 ; a]}=\frac{23}{168} h^{2}, \\
\sup _{f \in W_{\infty}^{2}[0 ; a]}\|f-S\|_{C[h / 2 ; h]}=\sup _{f \in W_{\infty}^{2}[0 ; a]}\|f-S\|_{C[a-h ; a-h / 2]} \leq \frac{7}{48} h^{2} .
\end{gathered}
$$


Д о к а з а т е л ь с т в о. Пусть сначала $x \in[0 ; h / 2]$. Действуя по теореме 3 , получаем

$$
S(x)-f(x)=\int_{0}^{x} K_{1,1}(x, t) f^{\prime \prime}(t) d t+\int_{x}^{h} K_{2,1}(x, t) f^{\prime \prime}(t) d t+\int_{h}^{2 h} K_{3,1}(x, t) f^{\prime \prime}(t) d t,
$$

где

$$
\begin{aligned}
& K_{1,1}(x, t)=\frac{x}{6 h}(6 h-7 t)-(x-t)=t-\frac{7 t x}{6 h}, \\
& K_{2,1}(x, t)=\frac{x}{6 h}(6 h-7 t), \\
& K_{3,1}(x, t)=\frac{x}{6 h}(t-2 h) .
\end{aligned}
$$

Имеем $K_{1,1}(x, t) \geq 0$ при $0 \leq t \leq x \leq h / 2, K_{3,1}(x, t) \leq 0$ при $h \leq t \leq 2 h, 0 \leq x \leq h / 2$. Функция $K_{2,1}(\cdot, t)$ меняет знак с плюса на минус в точке $t=6 h / 7$. Значит, для любой функции $f \in W_{\infty}^{2}[0 ; a]$ имеет место точная поточечная оценка

$$
\begin{gathered}
|f(x)-S(x)| \leq \int_{0}^{x} K_{1,1}(x, t) d t+\int_{x}^{6 h / 7} K_{2,1}(x, t) d t-\int_{6 h / 7}^{h} K_{2,1}(x, t) d t-\int_{h}^{2 h} K_{3,1}(x, t) d t \\
=\frac{11 x h}{21}-\frac{x^{2}}{2} \leq \max _{x \in[0 ; h / 2]}\left(\frac{11 x h}{21}-\frac{x^{2}}{2}\right)=\frac{23}{168} h^{2},
\end{gathered}
$$

которая достигается при $x=h / 2$ для функции $f$ такой, что $f^{\prime \prime}(t)=1$ при $0 \leq t \leq 6 h / 7$ и $f^{\prime \prime}(t)=-1$ при $6 h / 7<t \leq 2 h$. Ранее мы отмечали, что в силу "симметрии" построенного сплайна $S$ такая же точная оценка должна иметь место и на последнем полуотрезке $[a-h / 2 ; a]$.

Пусть теперь $x \in[h / 2 ; h]$. При этом имеем

$$
S(x)-f(x)=\int_{0}^{x} K_{1,2}(x, t) f^{\prime \prime}(t) d t+\int_{x}^{h} K_{2,2}(x, t) f^{\prime \prime}(t) d t+\int_{h}^{2 h} K_{3,2}(x, t) f^{\prime \prime}(t) d t,
$$

где

$$
\begin{aligned}
& K_{1,2}(x, t)=\frac{x}{6 h}(6 h-7 t)+\frac{2}{3 h^{2}}\left(x-\frac{h}{2}\right)^{2} t-(x-t), \\
& K_{2,2}(x, t)=\frac{x}{6 h}(6 h-7 t)+\frac{2}{3 h^{2}}\left(x-\frac{h}{2}\right)^{2} t, \\
& K_{3,2}(x, t)=(2 h-t)\left(\frac{2}{3 h^{2}}\left(x-\frac{h}{2}\right)^{2}-\frac{x}{6 h}\right) .
\end{aligned}
$$

Легко проверить, что $K_{1,2}(x, t)=\frac{t}{6 h^{2}}\left(4 x^{2}-11 x h+7 h^{2}\right) \geq 0$ при $0 \leq t \leq x, h / 2 \leq x \leq h$, $K_{3,2}(x, t)=\frac{2 h-t}{6 h^{2}}\left(4 x^{2}-5 x h+h^{2}\right) \leq 0$ при $h \leq t \leq 2 h, h / 2 \leq x \leq h$. Функция

$$
K_{2,2}(x, t)=\frac{1}{6 h^{2}}\left(6 x h^{2}-t\left(11 x h-4 x^{2}-h^{2}\right)\right)
$$

как функция от $t$ имеет единственный нуль

$$
t_{0}=t_{0}(x)=\frac{6 x h^{2}}{11 x h-4 x^{2}-h^{2}}
$$

на отрезке $[x ; h]$, причем в этом нуле меняет знак с плюса на минус. Пусть

$$
\Psi(x)=\int_{0}^{x} K_{1,2}(x, t) d t+\int_{x}^{t_{0}(x)} K_{2,2}(x, t) d t-\int_{t_{0}(x)}^{h} K_{2}(x, t) d t-\int_{h}^{2 h} K_{3,2}(x, t) d t .
$$


Из проведенного исследования для любой функции $f \in W_{\infty}^{2}[0 ; a]$ при любом $x \in[h / 2 ; h]$ выводим точное поточечное неравенство

$$
|f(x)-S(x)| \leq \Psi(x) .
$$

Для величины $\|\Psi\|_{C[h / 2 ; h]}$ получим оценку сверху. Заметим, что

$$
K_{2,2}(x, x) \geq\left|K_{2,2}(x, h)\right| \quad\left(\frac{h}{2} \leq x \leq h\right) .
$$

Поэтому

$$
\int_{x}^{t_{0}(x)} K_{2,2}(x, t) d t-\int_{t_{0}(x)}^{h} K_{2,2}(x, t) d t \leq \frac{(h-x) K_{2,2}(x, x)}{2}=\frac{5 x h-4 x^{2}-h^{2}}{12} \quad\left(\frac{h}{2} \leq x \leq h\right) .
$$

С другой стороны,

$$
\int_{0}^{x} K_{1,2}(x, t) d t-\int_{h}^{2 h} K_{3,2}(x, t) d t=\frac{\left(4 x^{2}-11 x h+7 h^{2}\right) x}{12 h} \quad\left(\frac{h}{2} \leq x \leq h\right) .
$$

Из (4.11)-(4.13) следует неравенство

$$
\Psi(x) \leq \frac{1}{12 h}\left(4 x^{3}-15 x^{2} h+12 x h^{2}-h^{3}\right) \quad\left(\frac{h}{2} \leq x \leq h\right) .
$$

Наибольшее значение правой части в последнем неравенстве равно $\frac{7}{48} h^{2}$ и достигается в точке $x=h / 2$. Таким образом, доказано неравенство

$$
\sup _{f \in W_{\infty}^{2}[0 ; a]}\|f-S\|_{C[h / 2 ; h]} \leq \frac{7}{48} h^{2} .
$$

Аналогичная оценка имеет место и на отрезке $[a-h ; a-h / 2]$.

Теорема 4 доказана.

В следующей теореме исследуется аппроксимация сплайнами, определенными формулой (4.1), класса функций $W_{\infty}^{3}=\left\{f: f^{\prime \prime} \in A C,\left\|f^{\prime \prime \prime}\right\|_{L_{\infty}(\mathbb{R})} \leq 1\right\}$.

Теорема 5. Для сплайнов $S(x)=S(f, x)$, определенных формулой (4.1), имеет место следующее равенство:

$$
\sup _{f \in W_{\infty}^{3}}\|f-S\|_{C}=M h^{3},
$$

где $M$ - некоторая положительная константа, причем 13/192 $\leq M \leq 7 / 32$.

Д о к а з а т е л ь с т в о. Любую функцию $f \in W_{\infty}^{3}$ можно представить в виде

$$
f(x)=f(j h)+\frac{f^{\prime}(j h)}{1 !}(x-j h)+\frac{f^{\prime \prime}(j h)}{2 !}(x-j h)^{2}+\frac{1}{2} \int_{j h}^{x}(x-t)^{2} f^{\prime \prime \prime}(t) d t .
$$

Для доказательства теоремы 5 можно, как и раньше, не ограничивая общности, считать, что $j=0$ и $x \in[0 ; h / 2]$. Поскольку сплайны вида (4.1) сохраняют квадратичные функции, то после элементарных преобразований получаем, что 


$$
\begin{aligned}
S(x)- & f(x)=\int_{-h}^{0} L_{1}(x, t) f^{\prime \prime \prime}(t) d t+\int_{0}^{x} L_{2}(x, t) f^{\prime \prime \prime}(t) d t \\
& +\int_{x}^{h} L_{3}(x, t) f^{\prime \prime \prime}(t) d t+\int_{h}^{2 h} L_{4}(x, t) f^{\prime \prime \prime}(t) d t,
\end{aligned}
$$

где

$$
\begin{gathered}
L_{1}(x, t)=\frac{(h+t)^{2} x(2 h-3 x)}{8 h^{2}} \quad(-h \leq t \leq 0), \\
L_{2}(x, t)=\frac{x}{8 h}\left[2(h-t)^{2}+\frac{x}{h}\left(h^{2}-2 h t+4 t^{2}\right)\right]-\frac{(x-t)^{2}}{2} \quad(0 \leq t \leq x), \\
L_{3}(x, t)=\frac{x}{8 h}\left[2(h-t)^{2}+\frac{x}{h}\left(h^{2}-2 h t+4 t^{2}\right)\right] \quad(x \leq t \leq h), \\
L_{4}(x, t)=-\frac{x^{2}(2 h-t)^{2}}{8 h^{2}} \quad(h \leq t \leq 2 h) .
\end{gathered}
$$

Очевидно, что $L_{1}(x, t) \geq 0(-h \leq t \leq 0), L_{4}(x, t) \leq 0(h \leq t \leq 2 h)$ при $0 \leq x \leq h / 2$. Исследование с помощью первой производной по аргументу $t$ показывает, что $L_{2}(x, t) \geq 0$ при $0 \leq t \leq x \leq h / 2$, а функция $L_{3}(x, t)$ как функция от $t$ меняет знак ровно один раз на отрезке $[x ; h]$, причем с плюса на минус. Из (4.15) следует, что нулем этой функции на указанном отрезке является число

$$
t_{3}=t_{3}(x)=\frac{2 h^{2}+3 h x-h \sqrt{5 x^{2}+2 x h}}{2 h+4 x} .
$$

Значит, из равенства (4.14) следует, что для любой функции $f \in W_{\infty}^{3}$ при любом $x \in[0 ; h / 2]$ имеет место точная оценка сверху

$$
\begin{aligned}
|S(x)-f(x)| & \leq \int_{-h}^{0} L_{1}(x, t) d t+\int_{0}^{x} L_{2}(x, t) d t+\int_{x}^{t_{3}(x)} L_{3}(x, t) d t \\
& -\int_{t_{3}(x)}^{h} L_{3}(x, t) d t-\int_{h}^{2 h} L_{4}(x, t) d t=g(x) .
\end{aligned}
$$

Из (4.15), (4.17) получаем, что

$$
\sup _{f \in W_{\infty}^{3}}\|f-S\|_{C}=\max _{x \in[0 ; h / 2]} g(x)=M h^{3}
$$

где $M$ - некоторая положительная константа. Вычислить точно $\max _{x \in[0 ; h / 2]} g(x)$ весьма затруднительно, принимая во внимание представление (4.16). Поэтому в теореме 5 мы ограничимся только получением оценок снизу и сверху для числа $M$. Для оценки снизу положим $x=h / 2$. Тогда $t_{3}=t_{3}(h / 2)=h / 2$ и

$$
\begin{gathered}
\left|S\left(\frac{h}{2}\right)-f\left(\frac{h}{2}\right)\right|=\int_{-h}^{0} L_{1}\left(\frac{h}{2}, t\right) d t+\int_{0}^{h / 2} L_{2}\left(\frac{h}{2}, t\right) d t-\int_{h / 2}^{h} L_{3}\left(\frac{h}{2}, t\right) d t-\int_{h}^{2 h} L_{4}\left(\frac{h}{2}, t\right) d t \\
=\frac{5 h^{3}}{192}+\frac{5 h^{3}}{384}+\frac{7 h^{3}}{384}+\frac{h^{3}}{96}=\frac{13}{192} h^{3} .
\end{gathered}
$$


Для получения оценки сверху заметим, что функции $L_{1}, L_{2}, L_{3}$ и $L_{4}$ являются многочленами второй степени по переменной $t$ и на соответствующих отрезках имеют место неравенства

$$
\left|L_{1}(x, t)\right| \leq \frac{h^{2}}{8}, \quad\left|L_{2}(x, t)\right| \leq \frac{9 h^{2}}{32}, \quad\left|L_{3}(x, t)\right| \leq \frac{5 h^{2}}{16}, \quad\left|L_{4}(x, t)\right| \leq \frac{h^{2}}{32} .
$$

Поэтому переходя к интегралам в неравенстве (4.17), получаем, что $M \leq 7 / 32$.

Теорема 5 полностью доказана.

3 а м е ч а н и е 2. Н. П. Корнейчук (см., например, [11, с. 322]) доказал, что для классических локальных параболических сплайнов $\widetilde{S}$ с равноотстоящими на $h$ узлами, сохраняющих квадратичные функции (определение и свойства см., например, в [8; 9, гл. IX]), имеет место равенство

$$
\sup _{f \in W_{\infty}^{3}}\|f-\widetilde{S}\|_{C}=\frac{3}{64} h^{3} .
$$

Сравнение этого результата с оценкой снизу для константы $M$ в теореме 5 показывает, что классические локальные параболические сплайны $\widetilde{S}$ приближают в равномерной метрике класс $W_{\infty}^{3}$ лучше, чем сплайны $S$ вида (4.1).

\section{Заключение}

Сравнение свойств двух рассмотренных в настоящей работе конструкций локальных параболических сплайнов показывает, что для прикладных исследований они примерно равноценны и могут быть получены из одной общей схемы. Сплайны Квасова обладают двумя преимуществами по сравнению со сплайнами Субботина, а именно, они сохраняют в отличие от сплайнов Субботина квадратичные функции и являются интерполяционными. Но по остальным рассмотренным свойствам (см. теоремы $A, B$ и теоремы 1-3) они уступают сплайнам Субботина. Интерполяционные сплайны не являются формосохраняющими (аналог теоремы $A$ для них не имеет места), и константа Лебега у них больше (т. е. они менее устойчивы к возмущению интерполяционных условий). На классе $W_{\infty}^{2}$ дважды дифференцируемых функций аппроксимативные свойства интерполяционных сплайнов также хуже, но, возможно, существует более оптимальный выбор их параметров из системы (3.1).

\section{СПИСОК ЛИТЕРАТУРЫ}

1. Субботин Ю.Н. Наследование свойств монотонности и выпуклости при локальной аппроксимации // Журн. вычисл. математики и мат. физики. 1993. Т. 33, № 7. С. 996-1003.

2. Шевалдин В.Т. Аппроксимация локальными параболическими сплайнами с произвольным расположением узлов // Сиб. журн. вычисл. математики. 2005. Т. 8, № 1. С. 77-88.

3. Шевалдин В.Т. Аппроксимация локальными сплайнами. Екатеринбург: УрО РАН, 2014. 198 с.

4. Кобылкин К.С. Применение формосохраняющих сплайнов для оценки плотности распределения земли между крестьянскими хозяйствами после реформы 1863 года // Вестн. Урал. ин-та экономики, управления и права. 2010. № 3. С. 94-99.

5. Гетманов В.Г. Методы вычисления аппроксимационных сплайновых функций для задач цифровой фильтрации // Изв. РАН. Теория и системы управления. 2016. № 5. С. 47-57.

6. Правдин С.Ф. Метод решения задачи реакции диффузии на несимметричной модели миокарда левого желудочка сердца // Тр. 47-й Междунар. молодеж. шк.-конф. "Современные проблемы математики и ее приложений" (CEUR-WS). 2016. T. 1662. С. 284-296.

7. Квасов Б.И. Интерполяция эрмитовыми параболическими сплайнами // Изв. вузов. Математика. 1984. № 5. C. $25-32$.

8. Lyche T., Schumaker L.L. Local spline approximation methods // J. Approx. Theory. 1975. Vol. 15, no. 4. P. 294-325.

9. Завьялов Ю.С., Квасов Б.И., Мирошниченко В.Л. Методы сплайн-функций. М.: Наука, 1980. $352 \mathrm{c}$. 
10. Квасов Б.И. Методы изогеометрической аппроксимации сплайнами. М.: Физматлит, 2006. 360 с. 11. Корнейчук Н.П. Сплайны в теории приближения. М.: Наука, 1984. 352 с.

Поступила 8.02.2019

После доработки 26.03.2019

Принята к публикации 1.04.2019

Субботин Юрий Николаевич

д-р физ.-мат. наук, чл.-корр. РАН, профессор

Институт математики и механики им. Н. Н. Красовского УрО РАН, г. Екатеринбург e-mail: yunsub@imm.uran.ru

Шевалдин Валерий Трифонович

д-р физ.-мат. наук, ведущий науч. сотрудник

Институт математики и механики им. Н. Н. Красовского УрО РАН, г. Екатеринбург e-mail: Valerii.Shevaldin@imm.uran.ru

\section{REFERENCES}

1. Subbotin Yu.N. Heritance of monotonisity and convexity properties under local approximation. J. Comp. Math. and Math. Physics, 1993, vol. 37, no. 7, pp. 996-1003 (in Russian).

2. Shevaldin V.T. Approximation by local parabolic splines with arbitrary knots. Sib. Zh. Vychisl. Mat., 2005, vol. 6, no 1, pp. 77-88 (in Russian).

3. Shevaldin V.T. Approksimatsiya lokal'nymi splainami [Local approximation by splines]. Ekaterinburg: Ural Branch of RAS Publ., 2014, 198 p.

4. Kobylkin K.S. The use of shape-preserving splines for estimating the density of distribution of land between peasant farms after the reform 1863. Vestnik Ural. Inst. Ekonomiki, Upravleniya i Prava, 2010, no 3, pp. 94-99 (in Russian).

5. Getmanov V.G. Evaluation of spline functions for digital filtering problems. J. Comput. Syst. Sci. Int., 2016, vol. 55, no. 5, pp. 725-734. doi: 10.1134/S1064230716040079.

6. Pravdin S.F. A method of solving reaction-diffusion problem on a non-symmetrical model of the cardiac left ventricle. CEUR Workshop Proc., vol. 1662. Proc. 47th Int. Youth School-Conf. "Modern Problems in Mathematics and its Applications", Yekaterinburg, Russia, 31 January - 6 February, 2016, pp. 284-296 (in Russian).

7. Kvasov B.I. Interpolation by parabolic Hermite splines. Soviet Math. (Izvestiya VUZ. Matematika), 1984, vol. 28 , no. 5 , pp. 29-37.

8. Lyche T., Schumaker L.L. Local spline approximation methods. J. Approx. Theory, 1975, vol. 15, no 4, pp. 294-325. doi: 10.1016/0021-9045(75)90091-X.

9. Zavyalov Yu.S., Kvasov B.I., Miroshnichenko V.L. Metody splain-funktsii [Methods of spline functions]. Moscow: Nauka Publ., 1980, 355 p.

10. Kvasov B.I. Metody izogeometricheskoi approksimatsii splainami [Methods of Shape-Preserving Spline Approximaton]. Moscow: Fizmatlit Publ., 2006, 360 p. ISBN: 5-9221-0733-X.

11. Korneichuk N.P. Splajny v teorii priblizheniya [Splines in approximation theory]. Moscow: Nauka Publ., $1984,352 \mathrm{p}$.

Received February 8, 2019

Revised March 26, 2019

Accepted April 1, 2019

Yurii Nikolaevich Subbotin, RAS Corresponding Member, Prof., Krasovskii Institute of Mathematics and Mechanics of the Ural Branch of the Russian Academy of Sciences, Yekaterinburg, 620108 Russia, e-mail: yunsub@imm.uran.ru .

Shevaldin Valerii Trifonovich, Dr.Phys.-Math. Sci., Krasovskii Institute of Mathematics and Mechanics of the Ural Branch of the Russian Academy of Sciences, Yekaterinburg, 620108, Russia. e-mail: Valerii.Shevaldin@imm.uran.ru .

Cite this article as: Yu. N. Subbotin, V. T. Shevaldin. A method of construction of local parabolic splines with additional knots, Trudy Instituta Matematiki i Mekhaniki URO RAN, 2019, vol. 25, no. 2, pp. 205-219. 